\title{
Surface-Enhanced Raman Spectroscopy: A New Modality for Cancer Imaging
}

\author{
Chrysafis Andreou*1, Sirish A. Kishore*1, and Moritz F. Kircher ${ }^{1-3}$ \\ ${ }^{1}$ Department of Radiology, Memorial Sloan Kettering Cancer Center, New York, New York; ${ }^{2}$ Center for Molecular Imaging and \\ Nanotechnology (CMINT), Memorial Sloan Kettering Cancer Center, New York, New York; and ${ }^{3}$ Department of Radiology, Weill \\ Cornell Medical College, New York, New York
}

\begin{abstract}
Although surface-enhanced Raman scattering (SERS) spectroscopy has traditionally been used as an in vitro analytic tool, in the past few years the first reports of the feasibility of in vivo imaging of cancer with biocompatible SERS probes have emerged. SERS imaging has great potential in the field of medical imaging because it offers several major advantages over other molecular imaging methods. Medical imaging using SERS nanoprobes can yield higher sensitivity and higher signal specificity than other imaging modalities, while also offering multiplexing capabilities that allow for unique applications. This article reviews the principles of SERS and highlights recent advances for in vivo cancer imaging. To present the abilities of this method as accurately as possible, the discussion is limited to studies in which the imaging data were confirmed by histological correlation.
\end{abstract}

Key Words: Raman; SERS; imaging; cancer; nanoparticles

J Nucl Med 2015; 56:1295-1299

DOI: 10.2967/jnumed.115.158196

When light interacts with matter, most photons are scattered elastically via the Rayleigh effect; that is, the emitted photons retain the same energy (hence frequency and wavelength) as the incident photons. However, a small fraction of the incident photons undergoes inelastic scattering. This process, termed Raman scattering or the Raman effect (1), manifests due to an energy exchange between the incident photon and the scattering substance. This energy exchange causes vibrations and stretching motions at particular atomic bonds of the scattering molecule. More importantly, it causes the emitted photons to have a frequency and wavelength different from the incident photons. Most often, the Raman-scattered light has lower energy, exhibiting a shift toward the red; this is referred to as Stokes scattering. However, if the scattered light has higher energy, it is referred to as anti-Stokes scattering. Different molecules will scatter light differently, resulting in a Raman spectrum so unique to the underlying substance that it has also been called a "Raman fingerprint." This principle forms the basis of Raman imaging and gives the method its unparalleled specificity, as illustrated in Figure 1A.

A major limitation of traditional Raman spectroscopy is that the fraction of Raman-scattered photons is very small $\left(\sim 1\right.$ in $\left.10^{7}\right)$. In biomedical settings, particularly when in vivo imaging with short

Received Apr. 5, 2015; revision accepted Jul. 9, 2015.

For correspondence or reprints contact: Moritz F. Kircher, Memorial Sloan Kettering Cancer Center, Department of Radiology, Center for Molecular Imaging and Nanotechnology (CMINT), Mortimer B. Zuckerman Research Center, 408 E. 69th St., Z-2062, New York, NY 10065.

E-mail: kircherm@mskcc.org

${ }^{*}$ Contributed equally to this work.

Published online Jul. 16, 2015.

COPYRIGHT (c) 2015 by the Society of Nuclear Medicine and Molecular Imaging, Inc. acquisition times is required, this intrinsic weakness of the Raman effect is prohibitive in most cases. However, several strategies have been used to amplify the number of Raman-scattered photons upon interaction with a substance of interest. The discovery of "surface-enhanced Raman scattering" (SERS) was perhaps the most crucial advancement in the field (2). SERS is a phenomenon in which the Raman intensity of a molecule is enhanced enormously (up to $10^{14}$-fold) when placed near a noble metal surface with high curvature, such as a silver or gold nanoparticle (3). This enhancement is attributed to a combination of contributing phenomena. The one most often cited is the existence of surface plasmon resonances caused by the collective oscillations of electrons in the conduction band of the nanoparticle. Another reported contributing effect is a charge-transfer resonance involving the transfer of electrons between the molecule and the conduction band of the metal. A third possible factor consists of resonances of the incident light within the molecule itself (3). In addition, numerous theoretical approaches have been invoked to maximize and control the influence of these factors, especially with regard to the surface plasmon resonances.

\section{SERS PROBE DESIGN CONSIDERATIONS FOR IN VIVO CANCER IMAGING}

A general scheme for SERS-based cancer imaging requires administration of the probes, which then accumulate in the cancerous tissue and allow for Raman imaging, as illustrated in Figure 1B. However, the fundamental complexities of SERS, in combination with the innate challenges of working with living systems, make it particularly difficult to develop SERS nanoparticles for in vivo cancer imaging. Optimal SERS enhancement of a Raman reporter molecule requires the use of nanoscale metal structures. Nanoparticles, however, generally do not accumulate in cancerous tissues in numbers comparable to those of small-molecule imaging agents used for PET or fluorescence imaging. Therefore, to enable in vivo tumor detection, the signal intensity of SERS nanoparticles must be especially high. This can be achieved by engineering the nanoparticles such that the metal-molecule system of the probe is in resonance with the incident laser wavelength. This method, termed "surface-enhanced resonance Raman scattering" (SERRS), gives rise to signals augmented by several additional orders of magnitude (3).

At the same time, the size and surface charge of SERS nanoparticles have a critical impact on their biodistribution and represent a limiting factor in the freedom of designing such probes for in vivo imaging. Gold nanoparticles, without a passivating shell, have the tendency to aggregate in vivo, which can lead to signal inhomogeneity. This, in turn, can result in attenuated signal or, occasionally, increased signal due to the formation of hot spots between the aggregated nanoparticles, potentially compromising the accuracy and interpretation of experimental data $(4,5)$. The placement of a silica or polyethylene glycol (PEG) coating on the surface of gold nanoparticles can prevent aggregation and also confers increased stability within a variety 


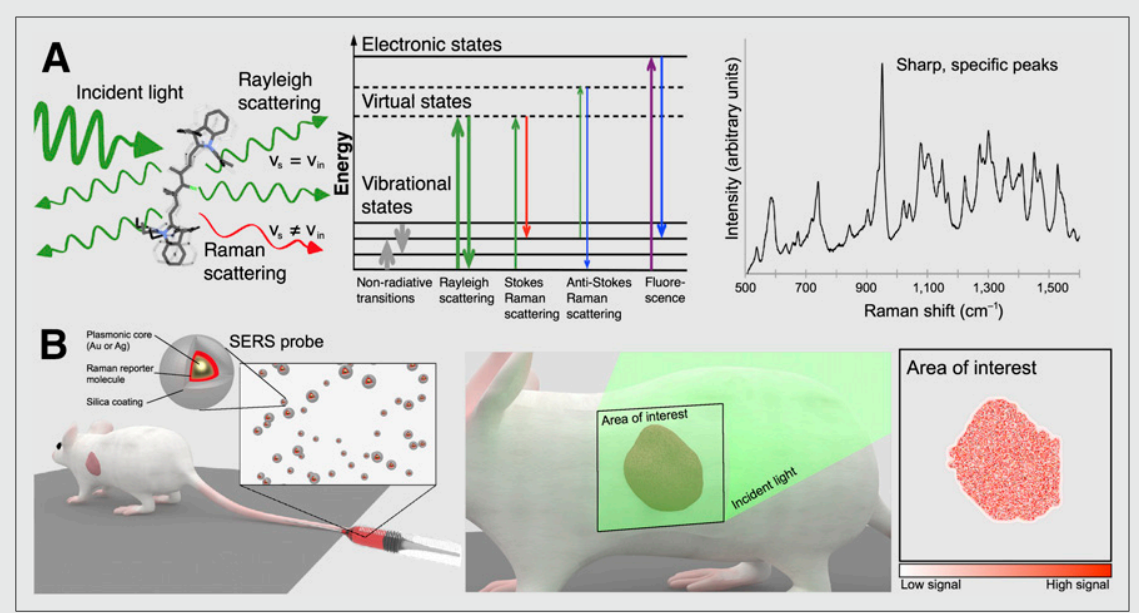

FIGURE 1. Principle of SERS nanoparticles for in vivo cancer detection. (A) Light scattering from molecule includes both Rayleigh and Raman scattered photons (left); schematic depiction of photon energy transitions during different types of light scattering shows that Rayleigh scattering is most common form of light scattering, with few photons undergoing Raman-related transitions (middle); Raman spectra exhibit peaks specific to molecular bond vibrations, showing sharp, high-intensity peaks characteristic of SERS probes (right). (B) SERS probes can be engineered to create strong SERS signals detectable in vivo. Although there are different routes of administration depending on tumor location and type, in most cases intravenous injection will be most desirable route (left). SERS probes typically consist of noble metal core (gold or silver), which enhances signal intensity via surface plasmon resonance effects, layer of Raman reporter molecule giving specific spectrum, and passivation layer (left inset). To enable cancer detection, SERS probes must accumulate in cancerous tissue, where they can be detected by their spectral signature upon interrogation with Raman imaging systems (middle). By color-coding pixels in acquired image where unique SERS spectrum of probe is detected, image of tumor is generated (right).

of microenvironments (6-9). However, this outer coating also introduces challenges, as the anchoring of silica to the gold surface may limit the number of Raman reporter molecules that can be incorporated onto the gold surface, taking a toll on the overall intensity. Finally, special considerations have to be given to the biocompatibility of such particles, and thus toxic materials must be avoided during their synthesis.

With these design constraints in mind, it is not surprising that, to date, only few studies have demonstrated the ability of SERS nanoparticles to allow in vivo imaging of cancer with correlative histopathologic validation. Our own group recently synthesized and tested a new SERRS nanoprobe (10) that is resonant in the near-infrared window, where optical penetration depth is maximized. This SERRS nanoprobe (Fig. 2A) has a star-shaped gold core demonstrating a localized surface plasmon resonance in the near-infrared window; a Raman reporter molecule that is in resonance with the detection laser $(785 \mathrm{~nm})$; and a biocompatible encapsulation method that, by avoiding the use of surface primers, allows efficient loading of the resonant Raman-reporter molecule at the gold surface. These SERRS nanoparticles-termed SERRS nanostars-were found to have a detection limit of $1.5 \mathrm{fM}$ using in vivo imaging settings, which represents an approximately 400-fold improvement over previous generations of nonresonant Raman nanoparticles (7). With the recent rational design of a new chalcogenopyrylium dye-based Raman reporter, this high sensitivity can be improved even further, resulting in detection limits in the attomolar range even with rapid in vivo imaging settings (11). Raman microscope scanners that are amenable to imaging of small animals in vivo are generally able to achieve a spatial resolution in the micrometer range (10). Although the imaging time increases with higher resolution, such improvements in SERRS nanoparticle signal amplification, as well as new generations of Raman scanners (12), are in turn able to decrease the scan time while interrogating even wider fields of view. A limitation of most nanoparticles coated with a silica shell is their relatively large size, which is above the cutoff for clearance through the renal glomerular membrane.
Efforts to integrate the Raman reporter into a polymer that is anchored directly to the gold core, without the need for silication, are allowing the synthesis of much smaller SERS particles (13). This fabrication strategy has the potential to produce SERS nanoparticles at sizes that would allow for renal clearance.

\section{NANOPARTICLE TARGETING: PASSIVE VERSUS ACTIVE}

In general, there are two major categories of strategies to achieve selective accumulation of nanoparticles in tumors, which also apply to SERS nanoparticles: passive and active targeting (4). Since the above-mentioned SERRS nanostars (10) do not require specific targeting moieties on the nanoparticle surface and yet allow robust tumor imaging, we conclude that their uptake depends on a property of cancer that is not unique to a specific type, subtype, or stage. It is already known that nanoparticles within a certain size range and surface charge accumulate specifically in cancer tissue but not in normal tissues. This uptake of nanoparticles without a specific targeting moiety on their surface is generally attributed to the so-called enhanced permeability and retention (EPR) effect (14-16). Initially described by Matsumara and Maeda, the EPR effect has been investigated quite extensively. It denotes a specific prolonged retention of macromolecules, including nanoparticles, in tumor tissue but not in healthy tissue (14-16). It has been reported to consist of two major pathophysiologic phenomena. The first is that tumor cell aggregates become dependent on blood flow supplied by neovasculature, which develops in response to vascular endothelial growth factor (VEGF) and other growth factors secreted by the tumor cells $(14,15)$. Tumor neovasculature is characterized by poorly aligned, defective endothelial cells with wide fenestrations and often lacks a smooth muscle layer. This allows nanoparticles to extravasate from the capillary bed into the tumor interstitium $(14,15)$. The second phenomenon is that tumor tissues lack effective lymphatic drainage, causing prolonged retention of macromolecules. The combination of these two phenomena leads to abnormal molecular and fluid transport dynamics for macromolecules. The EPR effect has been associated with virtually every cancer type (15). The fact that SERRS nanostars have enabled visualization of multiple different tumor types and precisely delineated tumor margins, microscopic tumor extensions, and metastases attests to the specificity of the EPR effect.

However, the EPR phenomenon is probably more complex than initially described, in that additional mechanisms may play a role in the delivery and retention of nanoparticles in tumors; we recently made the surprising observation that SERRS nanostars are also able to detect microscopic, premalignant lesions in genetic mouse models of pancreatic and prostate cancer. Because it is considered less likely that lymphatic drainage pathways are aberrant in small, premalignant lesions, this finding suggests the existence of an active cellular uptake mechanism. The process of macropinocytosis has recently gained the attention of both the nanotechnology and the cancer research communities. It is a unique mode of endocytosis in which extracellular fluid is internalized in a clathrin- and caveolin-independent manner that relies on actin-dependent ruffle formation. Interestingly, it has been shown that the driving oncogenic mutation in pancreatic cancer, $K$-Ras, stimulates macropinocytosis to 


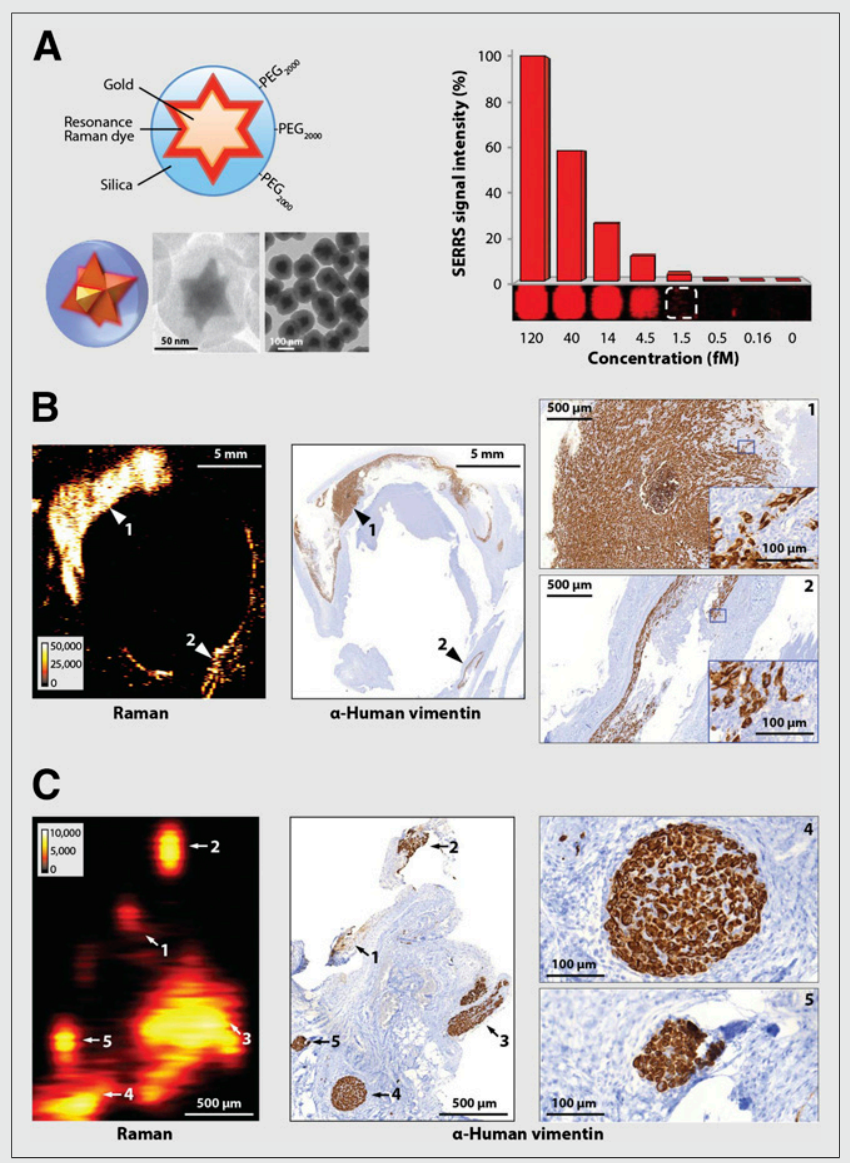

FIGURE 2. Imaging of cancer with microscopic precision using new generation of Raman nanoparticles. (A) Diagram, 3-dimensional rendering, and electron microscopy images of SERRS nanostars, which consist of star-shaped gold core surrounded by near-infrared Raman reporter and silica shell produced without use of surface primers (left). Phantom with decreasing concentrations of SERRS nanostars, acquired using in vivo imaging settings (right). Detection threshold is $\sim 1.5 \mathrm{fM}$. ( $B$ and C) After intravenous injection of only $30 \mathrm{fmol} / \mathrm{g}$, SERRS nanostars enable visualization of microscopic infiltration at tumor margins and regional satellite metastases. Experiments were performed on human dedifferentiated liposarcoma mouse model. SERRS images were acquired 16-18 $\mathrm{h}$ after injection, and signal intensity is displayed in counts/s. (B) Imaging of residual cancer in resection bed. SERRS image of resection bed was acquired after surgical excision of tumor bulk (left). Resection was guided by white light only, with surgeon blinded to SERRS images. Immunohistochemistry correlation confirmed that SERRS-positive signal (arrows 1 and 2) represented microscopic residual cancer at margins of resection bed (middle). Immunohistochemistry images on right are magnified views of areas indicated with arrows 1 and 2. (C) Imaging of regional satellite micrometastases. In different mouse bearing liposarcoma, SERRS image was acquired approximately $1 \mathrm{~cm}$ adjacent to visible margin of tumor (left). Multiple small foci of Raman signal are indicated (arrows 1-5). As confirmed by immunohistochemistry (middle), each of these 5 foci correlated with separate tumor cell cluster (vimentin+) as small as $100 \mu \mathrm{m}$ (micrometastases). Images on far right are magnified views of metastases labeled 4 and 5 . (Adapted with permission of (10).)

obtain amino acids from internalized proteins to support cancer cell metabolism (17). Because oncogenic mutation is an early event in cell transformation shared by many cancers, we reasoned that macropinocytosis might be an underlying mechanism for active SERRSnanoprobe internalization by malignant and premalignant cells. Indeed, we found that macropinocytosis inhibitors markedly reduced the intracellular uptake of SERRS nanoparticles in multiple cancer cell lines, supporting this theory (10). Other, unknown, factors may contribute to the enhanced permeability (EP) and retention (R) of the umbrella term EPR effect. This will require further investigations, and we expect their outcome to be highly variable depending on the exact size, geometry, surface chemistry, and surface charge of nanomaterials.

Active targeting, on the other hand, refers to the targeting of SERS probes to cancerous tissues using tissue-specific ligands. Theoretically, both passive and active strategies can be applied synergistically to optimize imaging of the tissue of interest, and this combined strategy is a topic under active investigation. As yet, there is no global consensus in the research community as to whether active targeting improves nanoparticle delivery over passive targeting. Our own preliminary data suggest that active targeting does not simply cause an amplification of probe accumulation within the tumor. Instead, there appears to be a redirection of the targeted probe to its ligand, resulting in accumulation that is less diffuse than EPR/macropinocytosis-based intratumoral probe accumulation. This does not necessarily mean that active targeting causes an overall higher concentration of the probe per tumor volume. Further studies are needed to elucidate the contribution of these factors to probe delivery.

\section{SERS-GUIDED SURGERY}

Because SERS nanoparticles for in vivo cancer detection can be designed to use a reporter molecule nonexistent in living subjects, the signal emitted by the nanoparticle serves as a Raman fingerprinta unique code that cannot be mistaken for intrinsic biologic background signal from the target tissue. This represents a major advantage over fluorescence imaging methods, with which it is often difficult to distinguish true signal from autofluorescence of the target tissue. Thus, SERS imaging has the potential to combine the signal specificity of a nuclear imaging method with the high resolution of optical imaging while boasting even higher sensitivity.

To our knowledge, the first demonstration of in vivo tumor resections using SERS imaging was reported in a mouse model of glioblastoma (7). In this study, gadolinium-DOTA-coated silica-gold nanoparticles were injected into the tail vein of an orthotopic glioblastoma mouse model, and sequential tumor resections were performed on live mice. Interestingly, SERS imaging was able to detect residual microscopic tumor in resection beds that was not detectable with the unaided eye. Because rapid wide-field SERS imaging devices for use in humans are not yet available, our group tested, in a separate study, whether a commercially available handheld Raman spectrometer could be used to guide brain tumor resections in a comparable fashion (18). We used a similar, albeit unimodal, type of SERS nanoparticles in the genetic RCAS/TVA mouse model, which spontaneously develops glioblastomas that closely mimic the human tumor biology. In a direct comparison between a Raman imaging device and a handheld Raman scanner, we found that the latter not only allowed real-time detection of tumor tissue but also was superior to static Raman imaging in that it could identify microscopic tumor cell clusters that were hidden behind normal brain tissue (18).

When attempting to image extracranial tumors with the SERS particles used in the brain tumor imaging studies mentioned above, we were unsuccessful. We concluded that although these particles were in fact already very sensitive (detection threshold, $600 \mathrm{fM}$ ) (7), this sensitivity was still not sufficient for imaging tumors that exhibit a lower EPR effect or less macropinocytotic activity than the glioblastoma mouse models. However, when we used the newly developed SERRS nanostars with their 400 -fold lower detection limit, we were able to image any tumor type we have tested so far. These tumors included breast cancer, prostate cancer, pancreatic cancer, and different types of sarcoma. The SERRS nanostars also allowed for very sensitive detection of residual microscopic tumor. As examples, Figure 2 shows detection of microscopic tumor infiltrations at the margins of 
the resected bulk tumor (Fig. 2B) and detection of locoregional micrometastases (Fig. 2C). In both cases, the detection of residual tumor surprised the operating surgeon, who had used conventional methods (white light illumination) and, blinded to the Raman imaging data, believed that he had performed complete tumor resections (10).

\section{MULTIMODAL SERS NANOPARTICLES}

One natural limitation of SERS imaging is that tissue penetration by the excitation light source is limited. Like other optical modalities, it is affected by absorption and scattering of photons with increasing depth, preventing whole-body imaging in humans. In addition to potentially improving the localization of SERS probes within deeper tissues, the development of multimodal SERS probes allows SERS to be combined with other, established, imaging technologies (19-22). This may enable the incorporation of Raman data into current diagnostic paradigms and facilitate clinical translation of SERS. For example, a triple-modality nanoparticle that combines SERS imaging with photoacoustic imaging and MR imaging has been developed and used successfully to monitor tumor localization and margins during the resection of malignant brain tumors (Fig. 3) (7). The particles could also be labeled with radiotracers via traditional techniques using chelator chemistry (23). Recently developed chelator-free methods for labeling silica with radiotracers (24) should further facilitate the design of combined PET/SERS nanoparticle probes. Such multimodal approaches could enable preoperative staging combined with intraprocedural guidance via SERS, in which both imaging techniques are detecting the same probe in the same tissues.

\section{SERS MULTIPLEXING}

SERS nanoparticles have the fundamental advantage of allowing multiplexing. As opposed to fluorescent dyes, which generally have only one broad emission peak, Raman reporters have spectra with multiple narrow peaks. This results in the high signal specificity of SERS imaging and also allows for discrimination between many different Raman reporters, and thus nanoparticles, without issues related to spectral overlap. This principle has been demonstrated with nontargeted SERS nanoparticles (25) and is expected to have great potential in the future in applications such as in vivo tumor marker expression profiling.

\section{TOWARD CLINICAL TRANSLATION}

Despite the unique and promising capabilities of SERS nanoparticles, some hurdles still stand in the way of clinical translation. The most important and most difficult obstacle to overcome will be obtaining the approval of the Food and Drug Administration for systemic injection of SERS nanoparticles into humans. SERS nanoparticles can be produced using relatively inert materials such as gold and silica, with only a trace of Raman reporter embedded within. Extensive cytotoxicity studies using a PEGylated gold-silica SERS nanoparticle (produced by Oxonica Materials, Inc.) have shown favorable results. The only reported side effect after tail vein injection of $9.6 \times 10^{10}$ nanoparticles (in $200 \mu \mathrm{L}$ of saline) into FVB mice was a mild inflammatory response in the liver, peaking at $24 \mathrm{~h}$ after nanoparticle injection (26). Such effects would be expected, as the liver is known to filter nanoparticles from the circulation. However, even if newer SERS nanoparticles were similar in size, geometry, and surface chemistry, such toxicity studies would have to be repeated for each new generation.

Another hurdle is that wide-field Raman scanners with real-time imaging capability are not yet available. These would be highly desirable, if not essential, for SERS imaging to be performed in most

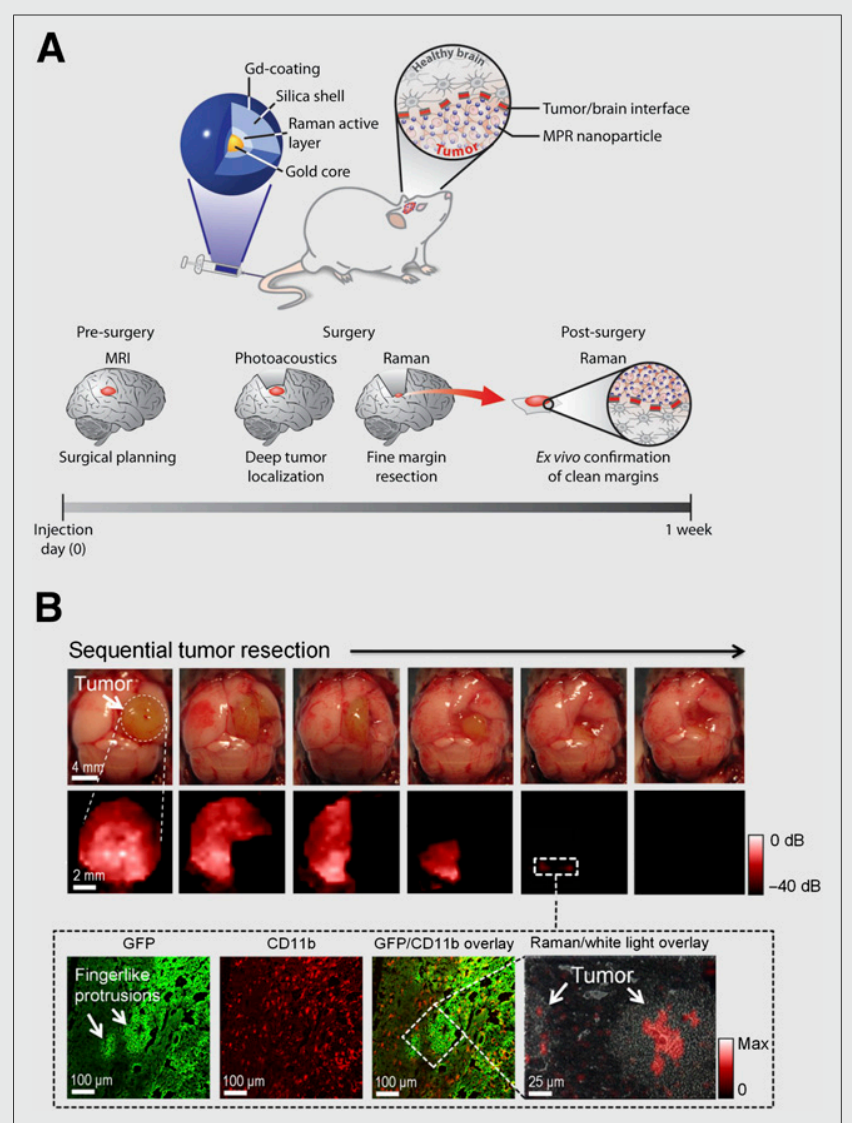

FIGURE 3. Multimodal SERS nanoparticles for pre- and intraoperative imaging of malignant brain tumors. (A) Triple-modality nanoparticle imaging concept. Nanoparticle is detectable by SERS, photoacoustic, and MR imaging (top). Nanoparticles are injected intravenously and home to brain tumor but not to healthy brain tissue. Because of stable, long-term internalization of nanoparticles within tumor tissue, preoperative MR imaging for staging and intraoperative imaging with SERS and photoacoustic imaging can be performed with single injection (bottom). (B) SERS-guided brain tumor resection in living mice. Intraoperative photographs show sequential resection steps, and SERS imaging shows corresponding residual tumor tissue at each resection step (top). After gross total resection, there is persistent SERS signal in normal-appearing resection bed, suggesting presence of residual cancer (white dashed square). Subsequent histological analysis of tissue containing these SERS-positive foci demonstrates residual cancer tissue invading surrounding normal brain (bottom). (Adapted with permission of (7).)

open surgical settings, and progress is being made toward this end (12). SERS imaging would also be well suited for endoscopic procedures. Raman endoscopes that fit into the instrument channel of a conventional white light endoscope have already been developed (25). Finally, advanced Raman detectors such as surface-enhanced spatially offset Raman scattering (SESORS) imaging systems are now in the prototype stage and have the potential to detect SERS probes several centimeters deep within the body (27). These advances in instrumentation should open many new avenues for the use of SERS nanoparticles once they are approved for human use (Fig. 4).

\section{CONCLUSION}

SE(R)RS nanoparticles have evolved into a new class of molecular imaging agents and in the past few years have shown promise in preclinical studies. The latest generations of these nanoparticles have major advantages over existing imaging agents, including much 


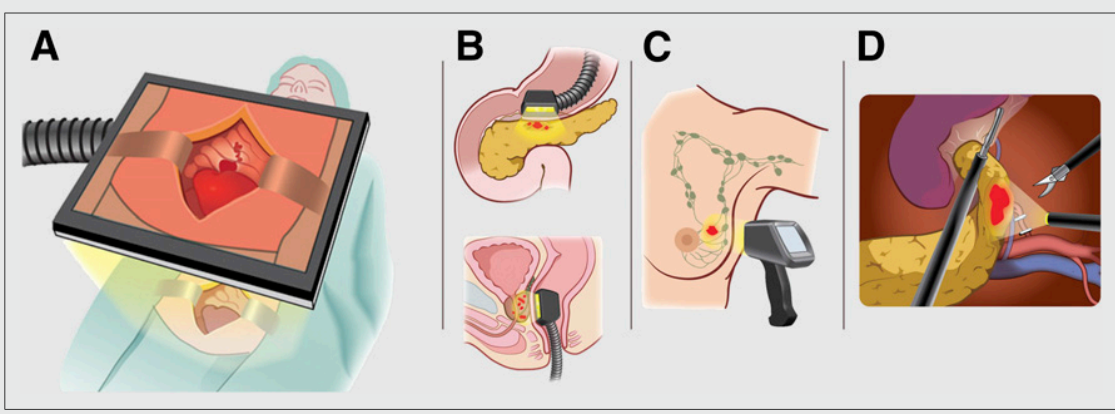

FIGURE 4. Potential clinical applications of SERS nanoparticles. (A) Raman imaging systems that allow wide-field-of-view coverage are currently in development (12) and could be used in operating room to visualize tumor margins, microscopic tumor infiltrations, and locoregional metastases. (B) Raman deeptissue imaging endoscopes using surface-enhanced spatially offset Raman spectroscopy (SESORS) technology (27) could be used for detection of cancers such as pancreatic (top) or prostatic (bottom) (C) Cancer types located within several centimeters of skin surface, such as breast cancer, could be detected noninvasively through skin with SESORS detectors. (D) Raman endoscopes (28) have potential to be used in endoscopic, laparoscopic, or robotically assisted tumor resections. (Expanded with permission of (10).)

higher sensitivity, nearly perfect signal specificity, and unparalleled multiplexing capabilities. With the hardware needed for use in humans already developed or in the pipeline, the largest hurdle toward clinical translation will be Food and Drug Administration approval of the nanoparticles themselves. However, because other types of gold and gold-silica nanoparticles for therapeutic purposes have already advanced into clinical trials (16), SERS nanoparticles should have a realistic chance for translation in the near future. The increased accuracy in visualizing the full extent of tumor spread provided by the SE(R)RS signal could increase the precision with which cancers can be resected or destroyed, be it via open surgical techniques or minimally invasive techniques used by interventional radiologists.

\section{DISCLOSURE}

Support was provided by the NIH (R01 EB017748 and K08 CA16396), the Damon Runyon Cancer Research Foundation (Innovation Award DRR-29-14), the Pershing Square Sohn Prize by the Pershing Square Sohn Cancer Research Alliance, the Dana Foundation Brain and Immuno-Imaging Grant and Dana Neuroscience Scholar Award, an RSNA Research Scholar Grant, a Geoffrey Beene Cancer Research Center Grant Award and Shared Resources Award, and grants from the MSKCC Center for Molecular Imaging and Nanotechnology (CMINT) and Technology Development Fund. No other potential conflict of interest relevant to this article was reported.

\section{ACKNOWLEDGMENTS}

We thank Stefan Harmsen, $\mathrm{PhD}$, and Matthew Wall, $\mathrm{PhD}$ (cand), for critical review of the manuscript and Ada Muellner, MS, and Ronald Blasberg, MD (all at MSKCC), for additional edits.

\section{REFERENCES}

1. Raman CV, Krishnan KS. A new type of secondary radiation. Nature. 1928;121: 501-502.

2. Zavaleta CL, Kircher MF, Gambhir SS. Raman's "effect" on molecular imaging. J Nucl Med. 2011;52:1839-1844.
3. Lombardi JR, Birke RL. The theory of surface-enhanced Raman scattering. J Chem Phys. 2012;136:144704.

4. Jokerst JV, Pohling C, Gambhir SS. Molecular imaging with surface-enhanced Raman spectroscopy nanoparticle reporters. MRS Bull. 2013;38(8).

5. Stranahan SM, Titus EJ, Willets KA. Discriminating nanoparticle dimers from higher order aggregates through wavelength-dependent SERS orientational imaging. ACS Nano. 2012;6:1806-1813.

6. Zavaleta CL, Smith BR, Walton I, et al. Multiplexed imaging of surface enhanced Raman scattering nanotags in living mice using noninvasive Raman spectroscopy. Proc Natl Acad Sci USA. 2009;106:13511-13516.

7. Kircher MF, de la Zerda A, Jokerst JV, et al. A brain tumor molecular imaging strategy using a new triplemodality MRI-photoacoustic-Raman nanoparticle. Nat Med. 2012;18:829-834.

8. Jokerst JV, Thangaraj M, Kempen PJ, Sinclair R, Gambhir SS. Photoacoustic imaging of mesenchymal stem cells in living mice via silica-coated gold nanorods. ACS Nano. 2012;6:5920-5930.

9. Chen YS, Frey W, Kim S, Kruizinga P, Homan K, Emelianov S. Silica-coated gold nanorods as photoacoustic signal nanoamplifiers. Nano Lett. 2011;11:348-354.

10. Harmsen S, Huang R, Wall MA, et al. Surface-enhanced resonance Raman scattering nanostars for high-precision cancer imaging. Sci Transl Med. 2015;7:271ra7.

11. Harmsen S, Bedics MA, Wall MA, Huang R, Detty MR, Kircher MF. Rational design of a chalcogenopyrylium-based surface-enhanced resonance Raman scattering nanoprobe with attomolar sensitivity. Nat Commun. 2015;6:6570.

12. Bohndiek SE, Wagadarikar A, Zavaleta CL, et al. A small animal Raman instrument for rapid, wide-area, spectroscopic imaging. Proc Natl Acad Sci USA. 2013;110: 12408-12413.

13. Iacono P, Karabeber H, Kircher MF. A "schizophotonic" all-in-one nanoparticle coating for multiplexed SE(R)RS biomedical imaging. Angew Chem Int Ed Engl. 2014;53:11756-11761

14. Maeda $H$. The link between infection and cancer: tumor vasculature, free radicals, and drug delivery to tumors via the EPR effect. Cancer Sci. 2013;104:779-789.

15. Maeda H, Nakamura H, Fang J. The EPR effect for macromolecular drug delivery to solid tumors: improvement of tumor uptake, lowering of systemic toxicity, and distinct tumor imaging in vivo. Adv Drug Deliv Rev. 2013;65:71-79.

16. Thakor AS, Gambhir SS. Nanooncology: the future of cancer diagnosis and therapy. CA Cancer J Clin. 2013;63:395-418.

17. Commisso C, Davidson SM, Soydaner-Azeloglu RG, et al. Macropinocytosis of protein is an amino acid supply route in Ras-transformed cells. Nature. 2013;497:633-637.

18. Karabeber H, Huang R, Iacono P, et al. Guiding brain tumor resection using surface-enhanced Raman scattering nanoparticles and a hand-held Raman scanner. ACS Nano. 2014;8:9755-9766.

19. Kircher MF, Gambhir SS, Grimm J. Noninvasive cell-tracking methods. Nat Rev Clin Oncol. 2011;8:677-688.

20. Kircher MF, Hricak H, Larson SM. Molecular imaging for personalized cancer care. Mol Oncol. 2012;6:182-195.

21. Kircher MF, Willmann JK. Molecular body imaging: MR imaging, CT, and US. Part I. principles. Radiology. 2012;263:633-643.

22. Kircher MF, Willmann JK. Molecular body imaging: MR imaging, CT, and US. Part II. Applications Radiology. 2012;264:349-368.

23. Deri MA, Zeglis BM, Francesconi LC, Lewis JS. PET imaging with ${ }^{89} \mathrm{Zr}$ : from radiochemistry to the clinic. Nucl Med Biol. 2013;40:3-14.

24. Shaffer TM, Wall MA, Harmsen S, et al. Silica nanoparticles as substrates for chelator-free labeling of oxophilic radioisotopes. Nano Lett. 2015;15:864-868.

25. Zavaleta CL, Garai E, Liu JT, et al. A Raman-based endoscopic strategy for multiplexed molecular imaging. Proc Natl Acad Sci USA. 2013;110:E2288-E2297.

26. Thakor AS, Luong R, Paulmurugan R, et al. The fate and toxicity of Ramanactive silica-gold nanoparticles in mice. Sci Transl Med. 2011;3:79ra33.

27. Stone N, Kerssens M, Lloyd GR, Faulds K, Graham D, Matousek P. Surface enhanced spatially offset Raman spectroscopic (SESORS) imaging: the next dimension. Chem Sci (Camb). 2011;2:776-780.

28. Garai E, Sensarn S, Zavaleta CL, et al. A real-time clinical endoscopic system for intraluminal, multiplexed imaging of surface-enhanced Raman scattering nanoparticles. PLoS One. 2015;10:e123185. 